\title{
REMOVABLE SINGULARITY SETS FOR ANALYTIC FUNCTIONS HAVING MODULUS WITH BOUNDED LAPLACE MASS
}

\author{
URBAN CEGRELL
}

\begin{abstract}
We prove that certain closed sets are removable singularity sets for analytic functions having modulus with bounded Laplace mass. As a special case, we find that every function which is analytic and with modulus having a harmonic majorant outside an analytic set extends analytically across this set.
\end{abstract}

1. Introduction. Denote by $B^{n}$ the unit ball in $\mathbf{C}^{n}$ and let $P$ be a closed subset of $B^{n}$. The purpose of this note is to prove a theorem which gives sufficient conditions on $P$ in order to insure that every analytic function $f$ on $B^{n} \backslash P$, such that $\Delta|f|^{q}$ has locally finite mass near $P$ for some $q>0$, has a (unique) extension to $B^{n}$. ( $\Delta$ denotes the Laplace operator.)

\section{A proposition.}

PROPOSITION. If $P$ has vanishing Newton capacity and if $\phi \geqslant 0$ is subharmonic on $B^{n} \backslash P$, then the following conditions are equivalent:

(i) $\varphi$ has a harmonic majorant on $B^{n} \backslash P$;

(ii) $\int_{B^{n} \backslash P} G(X, Y) \Delta \varphi(Y) Z+\infty$, where $G$ is Green's function for $B^{n}$;

(iii) $\varphi=\varphi_{1}-\varphi_{2}$, where $\varphi_{1}, \varphi_{2}$ are subharmonic and negative on $B^{n}$.

Proof. (i) $\Rightarrow$ (iii). If $0 \leqslant \varphi \leqslant h$ where $h$ is harmonic on $B^{n} \backslash P$, it follows that $\varphi-h$ and $-h$ have unique subharmonic extensions to $B^{n}$, since $P$ has vanishing Newton capacity (cf. Helms [6, p. 130]). Thus $\varphi=\varphi-h-(-h)$ on $B^{n} \backslash P$.

(iii) $\Rightarrow$ (ii). If $\varphi$ is subharmonic and negative on $B^{n}$, then

$$
\varphi(x)=-\int_{B^{n}} G(X, Y) \Delta \varphi(X)+h(X)
$$

by the Riesz decomposition theorem (cf. Helms [6, p. 116]). It follows that if $\varphi$ is the difference of two negative and subharmonic functions then (ii) holds.

(ii) $\Rightarrow$ (i). If $\psi(X)=\int_{B^{n} \backslash p} G(X, Y) \Delta \varphi(Y) Z+\infty$, then $\psi$ is superharmonic and nonnegative on $B^{n}$. Hence $\varphi+\psi$ is a majorant to $\varphi$ on $B^{n} \backslash P$, and since $\Delta \psi=-\Delta \varphi$, we have proved that (ii) implies (i), which completes the proof of the proposition.

Received by the editors January 1, 1981 and, in revised form, February 17, 1982 and September 10, 1982.

1980 Mathematics Subject Classification. Primary 32D20.

Key words and phrases. Removable singularity set, analytic function, harmonic majorant. 
REMARK 1. If $f=1 / z_{1}, P=\left\{z \in \mathbf{C}^{n}, z_{1}=0\right\}$, then $\Delta|f|^{q}=q^{2}\left|z_{1}\right|^{-(2+q)}$ so $\Delta|f|^{q}$ has infinite mass near $P$ which shows that (i) in the proposition cannot hold for $|f|^{q}$ (cf. Cima and Graham [4, §4]).

3. The exceptional sets. Let $E$ be a Borel set in $\mathbf{C}^{n}$. We define a set function $\gamma_{n}$ inductively (cf. Cegrell [2. p.11]). If $n=1$ then $\gamma_{1}=c$ where $c$ is the logarithmic capacity in $\mathbf{C}$. If $\gamma_{n-1}$ is defined on $\mathbf{C}^{n-1}$ we define $\gamma_{n}$ by

$$
\gamma_{n}(E)=c\left(\left\{z_{1} \in \mathbf{C} ; \gamma_{n-1}\left(\left\{\left(z_{2}, \ldots, z_{n}\right) ;\left(z_{1}, \ldots, z_{n}\right) \in E\right\}\right)>0\right\}\right) .
$$

Finally, we define $g_{n}(E)=\max _{\tau} \gamma_{n}(\tau E)$ where $\tau$ varies over the permutations of the coordinates. Our exceptional sets will be the sets $E$ with $g_{n}(E)=0$. Examples of such sets are pluripolar sets or, more generally, sets of vanishing $\Gamma$-capacity (cf. Ronkin [7] and Cegrell [3, p. 334]). Observe that if $g_{n}(E)=0$, then $E$ is of Lebesgue measure zero.

LemMA. Assume that $E$ is a nonempty set with $g_{n}(E)=0$. Then there is a point $z^{0}=\left(z_{1}^{0}, \ldots, z_{n}^{0}\right) \in E$ and an $m, 1 \leqslant m \leqslant n$, such that

$$
c\left(\left\{z \in \mathbf{C} ;\left(z_{1}^{0}, \ldots, z_{m-1}^{0}, z, z_{m+1}^{0}, \ldots, z_{n}\right) \in E\right\}\right)=0 .
$$

Proof. Induction. The case $n=1$ is clear so assume that $(*)$ holds in dimension $\leqslant n$. If $E \subset \mathbf{C}^{n+1}, g_{n+1}(E)=0$, and if no point of $E$ satisfies $(*)$, then we want to prove that $E=\varnothing$. Now, $c(Q)=0$, where

$$
Q=\left\{z_{1} \in \mathbf{C} ; \gamma_{n}\left(\left\{\left(z_{2}, \ldots, z_{n+1}\right) ;\left(z_{1}, \ldots, z_{n+1}\right) \in E\right\}\right)>0\right\} .
$$

So if $z_{1} \notin Q$ our induction assumption means that $\left(z_{1}, z_{2}, \ldots, z_{n+1}\right) \notin E$ for any point $\left(z_{2}, \ldots, z_{n+1}\right) \in \mathbf{C}^{n}$. Hence $E \subset Q \times \mathbf{C}^{n}$ so every point in $E$ satisfies (*), a contradiction, and the lemma is proved.

\section{Statement and proof of the theorem.}

Theorem. Assume that $P$ is closed in $B^{n}$ with $g_{n}(P)=0$. If $f$ is analytic on $B^{n} \backslash P$ and if $\Delta|f|^{q}$ has locally bounded mass near $P$, then $f$ extends to an analytic function on $B^{n}$.

Proof. Without loss of generality, we can assume that $\int_{B^{n} \backslash P} \Delta|f|^{q}<+\infty$. If $n=1$, then $P$ is of vanishing Newton capacity. By Proposition (iii) $|f|^{q}=\varphi_{1}-\varphi_{2}$, where $\varphi_{1}$ and $\varphi_{2}$ are subharmonic on $B^{1}$. It follows that $f \in L_{\text {loc }}^{s}\left(B^{1}\right) \forall s>0$, then by Carleson [1, p. 73] or Harvey and Polking [5, Theorem 1.1], $f$ extends to an analytic function.

Assume now that the theorem is proved for dimension $\leqslant n$ and let $P$ be closed in $B^{n+1}$ with $g_{n+1}(P)=0$. Let $0 \leqslant \chi_{\nu} \in C_{0}^{\infty}\left(B^{n+1} \backslash P\right)$ be an increasing sequence of functions tending to one.

Put

$$
\tau_{\nu}\left(z_{m}\right)=\int_{\mathbf{C}^{n}}|f|^{q} \sum_{s \neq m} \frac{\partial^{2} \chi_{\nu}}{\partial z_{s} \partial \bar{z}_{s}}\left(d z_{m}\right)^{\wedge},
$$


where $\left(d z_{m}\right)^{\wedge}$ is the Lebesgue measure in $\mathbf{C}^{n}$. Since $|f|^{q}$ is plurisubharmonic on $B^{n+1} \backslash P, \tau_{\nu}$ is an increasing sequence and

$$
\int_{\mathbf{C}} \tau_{\nu}\left(z_{m}\right) d z_{m} \leqslant \int_{B^{n+1} \backslash P} \Delta|f|^{q}<+\infty
$$

Hence

$$
\lim _{\nu \rightarrow+\infty} \tau_{\nu}\left(z_{m}\right)<+\infty, \quad \text { a.e. }\left(d z_{m}\right)
$$

so by the induction assumption,

$$
\left(z_{1}, \ldots, z_{m-1}, z_{m+1}, \ldots, z_{n+1}\right) \mapsto f\left(z_{1}, \ldots, z_{n+1}\right)
$$

extends to an analytic function for every $z_{m}$ outside a set of planar Lebesgue measure zero.

Put

$$
\varphi(z)=\varlimsup_{\substack{z^{\prime} \overrightarrow{z^{\prime}} \\ z^{\prime} \in B^{n} \backslash P}}\left|f\left(z^{\prime}\right)\right|, \quad z \in B^{n+1},
$$

and

$$
W=\left\{z \in B^{n+1} ; \varphi(z)<+\infty\right\} .
$$

Then $W$ is open, contains $B^{n+1} \backslash P$ and $f$ has an analytic extension to $W$, since the function $f$ defines a locally integrable distribution on $W$, and if $\chi \in C_{0}^{\infty}(W), i \neq j$, then

$$
\int_{\mathbf{C}^{n+1}} f \frac{\partial \chi}{\partial \bar{z}_{i}}=\int_{\mathbf{C}} d z_{j} \int_{\mathbf{C}^{n}} f \frac{\partial \chi}{\partial \bar{z}_{i}}\left(d z_{j}\right)^{\wedge}=0
$$

by $(* *)$.

It remains to prove that $W=B^{n+1}$. If not, then $P^{\prime}=B^{n+1} \backslash W$ is closed in $B^{n+1}$, nonempty and contained in $P$. Hence, by the lemma, there is a point $z^{0} \in P^{\prime}$ with property (*). Since $P^{\prime}$ is closed and since $c(Q)=0$, where

$$
Q=\left\{z \in \mathbf{C} ;\left(z_{1}^{0}, \ldots, z_{m-1}^{0}, z, z_{m+1}^{0}, \ldots, z_{n+1}^{0}\right) \in P^{\prime}\right\}
$$

we can choose $r>0$ so that $T \subset \subset B^{n+1} \backslash P^{\prime}=W$, where

$$
T=\left\{\left(z_{1}^{0}, \ldots, z_{m-1}^{0}, z, z_{m+1}^{0}, \ldots, z_{n+1}^{0}\right) ;\left|z-z_{m}^{0}\right|=r\right\} .
$$

Now let $z^{\nu} \in W$ be a sequence of points tending to $z^{0}$ so that $\left|f\left(z^{\nu}\right)\right| \rightarrow+\infty$, $\nu \rightarrow+\infty$. Since $|f|$ is continuous on $W$ we can, by $(* *)$, assume that $z \mapsto$ $f\left(z_{1}^{\nu}, \ldots, z_{m-1}^{\nu}, z, z_{m+1}^{\nu}, \ldots, z_{n+1}^{\nu}\right)$ is analytic near $\left|z-z_{m}^{\nu}\right| \leqslant r$, and the maximum principle gives

$$
\left|f\left(z^{\nu}\right)\right| \leqslant \sup _{\left|z-z_{m}^{\nu}\right|=r}\left|f\left(z_{1}^{\nu}, \ldots, z_{m-1}^{\nu}, z, z_{m+1}^{\nu}, \ldots, z_{n+1}^{\nu}\right)\right| .
$$

Hence, $\varlimsup_{\nu \rightarrow+\infty}\left|f\left(z^{\nu}\right)\right| \leqslant \sup _{\eta \in T}|f(\eta)|<+\infty$, which is a contradiction, so $P^{\prime}$ is empty, which proves the theorem. 


\section{Concluding remarks.}

REMARK 2. Since every analytic set is of vanishing Newton capacity and satisfies $g_{n}=0$, the theorem together with the proposition gives a generalization of Theorems $A$ and B in Cima and Graham [4].

REMARK 3. There are many variations of the theorem: Assume that $P$ is closed in $B^{n}$ with $g_{n}(P)=0$. If $f$ is analytic on $B^{n} \backslash P$ and if there is a $q>0$ and an $n$-subharmonic function $\psi$ on $B^{n} \backslash P$ such that $|f|^{q} \leqslant-\psi$ on $B^{n} \backslash P$, then $f$ extends to an analytic function on $B^{n}$.

\section{REFERENCES}

1. L. Carleson, Selected problems on exceptional sets, Van Nostrand, Princeton, N. J., 1967.

2. U. Cegrell, Construction of capacities on $\mathbf{C}^{n}$, U.U.D.M. 1978:1; Lecture Notes in Math., vol. 822. Springer-Verlag, Berlin and New York.

3. Removable singularities for plurisubharmonic functions and related problems, Proc. London Math. Soc. 36 (1978), 310-336.

4. J. A. Cima and I. R. Graham, On the extension of holomorphic functions with growth conditions across analytic subvarieties, Michigan Math. J. 28 (1981), 24l-256.

5. R. Harvey and J. Polking, Extending analytic objects, Comm. Pure Appl. Math. 28 (1975), 7()1-727.

6. L. L. Helms, Introduction to potential theory, Wiley, New York, 1969.

7. L. I. Ronkin, Introduction to the theory of entire functions of several variables, Transl. Math. Mono., vol. 44, Amer. Math. Soc., Providence, R. I., 1974.

Department of Mathematics, McGill University, Montraal, Canada

Current address: Department of Mathematics, Uppsala University, Uppsala, Sweden 